\title{
Article
}

Arq Neuropsiquiatr 2010;68(2):168-173

\section{Epilepsy in patients with psychogenic non-epileptic seizures}

\author{
Renato Luiz Marchetti ${ }^{1}$, Daniela Kurcgant ${ }^{1}$, José Gallucci-Neto', \\ Mary Ann Von Bismark', Lia Arno Fiore²
}

\begin{abstract}
The aim of this study was to evaluate the frequency of epilepsy in patients who presented psychogenic non-epileptic seizures (PNES). The evaluation was carried out during intensive VEEG monitoring in a diagnostic center for epilepsy in a university hospital. The difficulties involved in reaching this diagnosis are discussed. Ninety-eight patients underwent intensive and prolonged video-electroencephalographic (VEEG) monitoring; out of these, a total of 28 patients presented PNES during monitoring. Epilepsy was defined as present when the patient presented epileptic seizures during VEEG monitoring or when, although not presenting epileptic seizures during monitoring, the patient presented unequivocal interictal epileptiform discharges. The frequency of epilepsy in patients with PNES was 50\% (14 patients). Our findings suggest that the frequency of epilepsy in patients with PNES is much higher than that of previous studies, and point out the need, at least in some cases, for prolonging the evaluation of patients with PNES who have clinical histories indicating epilepsy. Key words: PNES, psychogenic non-epileptic seizures, pseudoseizures, epilepsy.
\end{abstract}

\section{Epilepsia em pacientes com crises não epilépticas psicogênicas}

\section{RESUMO}

O objetivo deste estudo foi avaliar a frequência de epilepsia em pacientes que apresentaram crises não epilépticas psicogênicas (CNEP). Isto foi realizado durante monitoração intensiva por video-EEG num centro diagnóstico de epilepsia em um hospital universitário. As dificuldades envolvidas para se chegar a este diagnóstico são discutidas. Noventa e oito pacientes foram submetidos a monitoração intensiva por video-EEG; 28 destes pacientes apresentaram CNEP durante a monitoração. Epilepsia foi considerada presente quando o paciente apresentou crises epilépticas durante a avaliação pelo video-EEG ou quando, apesar da não ocorrência de crises epilépticas durante a avaliação, descargas epilépticas interictais inequívocas estavam presentes. A frequência de epilepsia em pacientes com CNEP foi 50\% (14 pacientes). Nossos achados sugerem que a frequência de epilepsia em pacientes com CNEP é maior do que a apresentada em estudos anteriores e apontam para a necessidade de, ao menos em alguns casos, prolongar a avaliação de pacientes com CNEP, mas com história clínica sugestiva de epilepsia.

Palavras-chave: CNEP, crises não epilépticas psicogênicas, pseudocrises, epilepsia.

\section{Correspondence \\ Renato Luiz Marchetti \\ Rua Cayowaa 1236 / 61 \\ 05018-001 São Paulo SP - Brasil \\ E-mail: rlmarche@bighost.com.br}

Received 6 September 2009

Received in final form 9 October 2009

Accepted 21 October 2009
Non-epileptic seizures (NES) are recurring seizures, attacks or spells that may be mistaken for epilepsy due to the similarity of behavioral manifestations, but that are not caused by abnormal cerebral discharges. NES seizures may be physiological or psychogenic in origin (PNES), and PNES is the most frequent type. Stud- ies on the prevalence of PNES have shown different results of significant clinical importance. Among outpatients with epilepsy, 5 to $33 \%$ presented PNES ${ }^{1,2}$. Among patients hospitalized for evaluation of refractory epilepsy, 10 to $58 \%$ presented $\mathrm{PNES}^{3-5}$. The prevalence in the general population is estimated to be between 2 and 33/100000 . 
The only two epidemiological studies carried out on the subject of PNES show that the annual incidence of PNES in the general population is 1.4 to $3 / 100000^{6,7}$. According to $\mathrm{Gates}^{8}$, the differences obtained in these figures may be explained by the methodology used for diagnosis. PNES and its myriad presentations have challenged and confused psychiatrists and neurologists for several centuries. Since the 80's, the knowledge of PNES has grown as a consequence of the growing use of intensive video-electroencephalographic (VEEG) monitoring. VEEG is currently considered to be the "gold standard" for the correct diagnosis of $\mathrm{NES}^{9,10}$.

This diagnosis is complicated by the possible coexistence of epilepsy and PNES. Reports show that 5.3 to $73 \%$ of patients with PNES also present epilepsy ${ }^{3,11-16}$. This variability in different studies may reflect several methodological characteristics and is an important diagnostic issue.

The aim of this study was to assess the frequency of epilepsy in patients who presented PNES during intensive VEEG monitoring in a diagnostic center for epilepsy in a university hospital in São Paulo, Brazil. The difficulties involved in reaching this diagnosis have also been discussed.

\section{METHOD}

From 2003 to 2006, 98 patients underwent intensive and prolonged VEEG monitoring in the Neurophysiology Laboratory of the Institute and Department of Psychiatry, University of São Paulo School of Medicine. Out of these, a total of 28 patients presented PNES during monitoring. These patients, usually referred by other neurological centers, were evaluated for the following reasons: investigation for epilepsy surgery, differential diagnosis of epilepsy refractory to antiepileptic drugs (AED), or for suspected PNES. All patients were hospitalized in the VEEG unit for a variable period of time for simultaneous recording of behavior and EEG activity, thereby allowing observation and identification of registered seizures. The evaluation was carried out using the Ceegraph PTI digital equipment (Biologic Systems Corp), with version 6.72 .06 software. Electrodes were placed according to the international 10-20 electrode placement system with the addition of zygomatic and electrocardiogram electrodes. At baseline, the EEG (awake and sleep) was obtained, and activating procedures (hyperventilation and photostimulation) were carried out before changes in the AED regimen. For all patients for whom monitoring was recommended due to suspected PNES, as well as for all those hospitalized for other reasons but in whom spontaneous PNES were registered, the following sequential PNES induction protocol was carried out: simple suggestion, suggestive interview, induction with hypnotic or post-hypnotic suggestion and intravenous placebo infusion (saline solution). The sequence was interrupted as soon as a PNES were obtained. Following these procedures, AEDs were gradually decreased and records continued for observation of events and/or electroencephalographic changes, during periods considered sufficient to allow diagnosis.

For the completion of this study, for all patients in whom PNES was previously suspected or in whom either spontaneous or provoked PNES were obtained, the investigation protocol was prolonged with the purpose of recording discharges or epileptic seizures after complete AED withdrawal. This led to a hospitalization period lasting typically three weeks (range from 1 to 6 weeks).

An event produced by the PNES induction protocol was defined as PNES when neither epileptiform discharges nor ictal epileptiform EEG patterns were obtained before, during or after the event. A spontaneous event occurring at any moment was defined as an ES when accompanied by unequivocal discharges or ictal EEG patterns before, during or soon after its occurrence. A spontaneous event not fulfilling these conditions was only defined as PNES when its characteristic semiology was similar to PNES provoked by suggestive induction. All events recorded were analyzed and shown to family members so that they could confirm whether these were equal to those presented by patients in their daily lives.

Some patients may, in extreme situations, such as prolonged intensive monitoring by VEEG, present isolated PNES without constituting a real and significant clinical problem ${ }^{17}$. Only the cases in which PNES occurring during VEEG was validated by clinical history (clinical validation) and by exhibiting the video to an external observer close to the patient (observer validation) were thus considered to be a real clinical problem. The investigation for PNES was then considered to be positive and the condition was defined as a PNES disorder (PNESD).

Epilepsy was defined when the patient presented epileptic seizures during VEEG monitoring or when, although not presenting epileptic seizures during monitoring, the patient presented unequivocal interictal epileptiform discharges (sharp waves, spikes or spike-wave complexes). Cases were also considered to be epilepsy when such occurrences were validated by consistent information suggestive of epileptic seizure by clinical history obtained with the patient or by an external observer close to the patient (clinical validation). Benign variants were not considered to be epileptiform conditions. When the presence of interictal epileptiform discharges was not corroborated by clinical validation, epilepsy was not defined as present.

For every patient, the following diagnostic possibilities were considered: 
1. Concerning epilepsy: current diagnosis of epilepsy absent; in remission; in remission under treatment; mildly, moderately or severely active.

2. Concerning PNESD: current diagnosis of PNESD absent; in remission; in remission under treatment; mildly, moderately or severely active. The mental disorder presenting as PNESD was defined.

3. Concerning both diagnoses (epilepsy and PNES), the following levels of conviction were obtained by diagnostic investigation: presumed, probable or definitive.

4. Psychiatric comorbidities, possibly associated with epilepsy, PNESD or both, were defined.

Patients underwent neurological, psychiatric, imaging (magnetic resonance imaging, interictal SPECT and possibly ictal SPECT) and neuropsychological evaluations. After reaching the diagnoses, these were communicated together with a referral for the appropriate treatments.

The diagnosis of seizure was defined according to the International Classification of Epileptic Seizures ${ }^{18}$. Diagnoses of epileptic syndromes were defined according to the International Classification of Epileptic Syndromes ${ }^{19}$.

Psychiatric evaluation was carried out in turns by one of three psychiatrists (JGN, MAVB and RLM) with training and experience in the neuropsychiatry of epilepsy. The evaluation was performed by means of open clinical interviews during the VEEG monitoring period. All cases evaluated were reviewed together by the three professionals, and the diagnoses were reached by consensus.

Neurological evaluation was carried out by epileptologists with ample experience in VEEG monitoring (LAF).

An information statement, in which all the procedures of VEEG monitoring were explained, was given to and discussed with the patients and their relatives before the beginning of each investigation. All patients provided written informed consent and the protocol was in agreement with the institutional research ethics board.

\section{RESULTS}

Out of 28 patients, 22 (78.6\%) were referred for intensive VEEG monitoring for suspected PNES. Six patients (21.4\%) were referred for other reasons: two (7.1\%) for pre-surgical evaluation and four (14.3\%) for diagnosis of an epileptic syndrome refractory to clinical treatment.

The results are presented in the Table. Out of $28 \mathrm{pa}-$ tients, 26 (93\%) were female. The mean age was 37 years (range from 19 to 62, median=38, standard deviation=10).

The patients were divided into three groups: $14 \mathrm{pa}-$ tients presented PNES during VEEG monitoring, evidence of PNES validated by clinical and observer validation and absence of epilepsy (PNESD group); 13 patients presented PNES during VEEG monitoring, evidence of PNESD and epilepsy (PNESD/E group); one patient (case 28) presented PNES during VEEG monitoring, but no evidence of PNESD (PNES was not clinically or observer validated), and presented epilepsy. Therefore, the frequency of epilepsy in patients with PNES was equal to $50 \%$ (14 patients).

The Table shows detailed data on patients population. Five patients (18\%) presented epileptic seizures (ES) during monitoring. Nine patients (32\%) did not present ES during VEEG monitoring, but presented unequivocal epileptiform discharges corroborated by clinical validation, allowing the diagnosis of epilepsy. One patient (case 4), although presenting unequivocal epileptiform discharges, did not receive the diagnosis of epilepsy, due to the absence of ES during VEEG monitoring, and without corroborative clinical validation.

Out of the 14 patients with epilepsy, five presented partial complex seizures, three had secondarily generalized seizures, two had absence seizures (of whom one also had primarily generalized tonic-clonic seizures), one had myoclonic seizures and five presented unclassified seizures. Therefore, eight patients presented partial epilepsy (five with temporal lobe and three with frontal lobe epilepsy), three had generalized epilepsy (one with juvenile myoclonic epilepsy, one with childhood absence epilepsy and one with juvenile absence epilepsy), and three were considered to be undetermined epilepsies. Three of the 14 patients with epilepsy were in remission, five were in remission under treatment and six presented active epilepsy.

In only two of the 28 patients with PNES was the diagnoses of PNESD during VEEG considered to be presumed, because PNES could not be fully validated, due to the similarities between PNES and ES. In one patient (case 28), the diagnosis of PNES was not confirmed, since PNES was not clinically nor observer validated. In all other cases, the diagnoses of PNESD was considered to be definitive.

In 11 out of the 14 patients with epilepsy, the diagnosis was considered to be definitive. In only three of these patients was the diagnosis considered to be probable. In these cases, the patients did not present ES during VEEG, but presented unequivocal interictal epileptiform activity, while the clinical validation left doubts as to seizure semiology or clinical course.

\section{DISCUSSION}

Surely, one of the clinical situations that produce the greatest polemics is the association of epilepsy and PNES. The prevalence of epilepsy in patients with PNES has been estimated as ranging from 5.3 to $73 \%^{3,11-16}$. This variability in different studies may reflect several methodological characteristics, such as the different inclusion criteria when determining epilepsy and PNES, the presence or not of ictal/interictal EEG abnormalities, the diagnostic environment (inpatient or outpatient), the pres- 


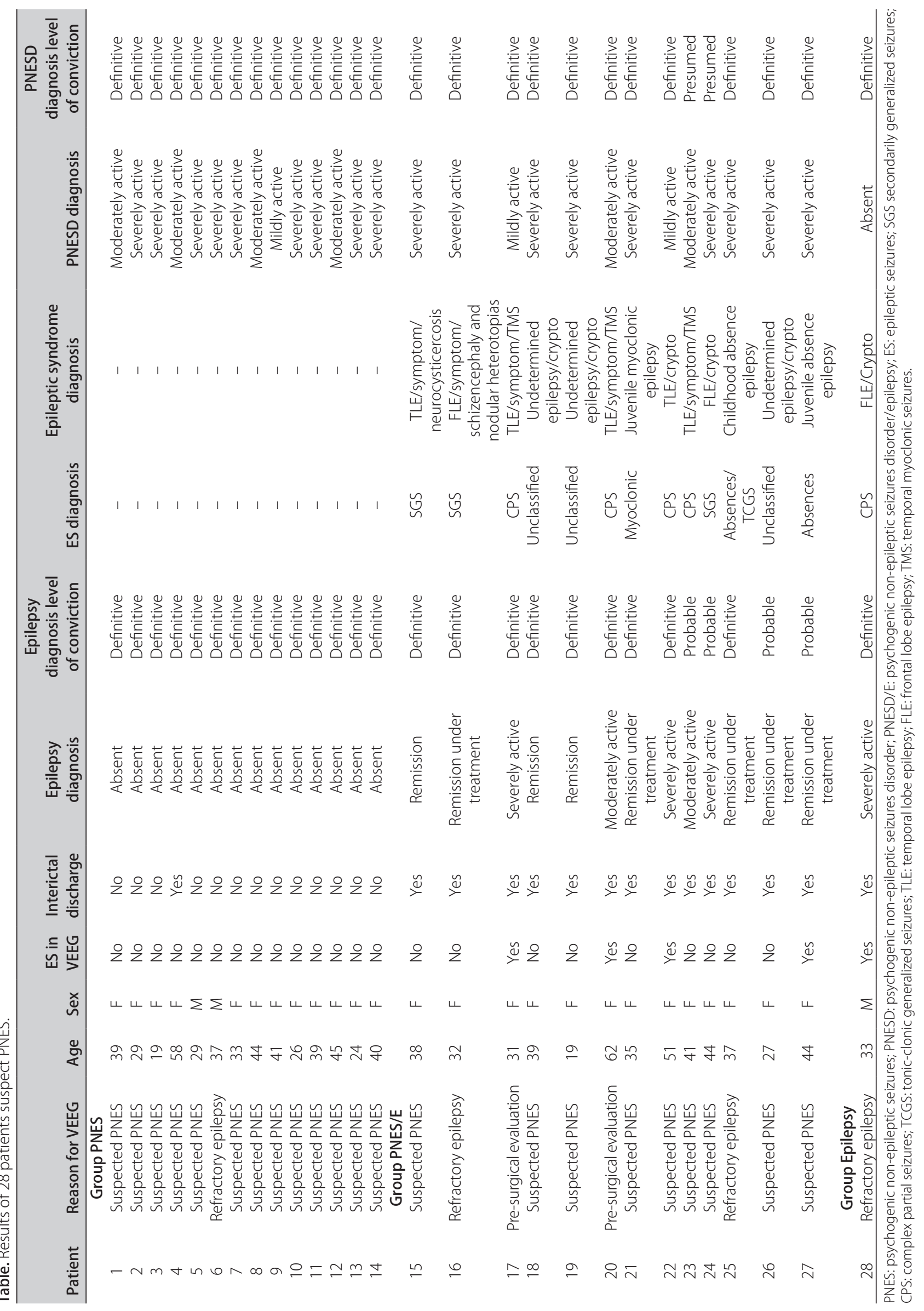


ence of prolonged monitoring by VEEG, the monitoring duration and the sample size.

This diagnostic issue is extremely important. In the first place, omission of the PNES diagnosis may be very harmful and damaging to patients. Martin et al. ${ }^{20}$ estimated that the lifetime costs borne by a person with PNES, in diagnostic tests, procedures and treatments would be around US\$100,000. They also calculated that US\$100 to 900 million are spent yearly in the USA on the PNES patient population. Several studies have shown that early and appropriate diagnosis of PNES, followed by adequate treatment, may lead to remission in 19 to $52 \%$ of cases, or to improvement in 75 to $95 \%$ of cases. Therefore, a significant reduction in the use of healthcare systems and in costs is involved $\mathrm{d}^{20-23}$. PNES leads to severe social and psychological consequences. These patients and their families face the same problems as patients with epilepsy: stigmatization, poor schooling, unemployment, difficulties in interpersonal relationships and social exclusion ${ }^{24}$. From the medical point of view, patients are exposed to iatrogenic procedures, such as the use of high doses of $\mathrm{AED}^{25}$, venous punctures, intravenous AED, and orotracheal intubation ${ }^{26}$. Moreover, the rate of comorbidity with depressive and anxiety disorders is high ${ }^{14,21}$, and the quality of life of these patients is worse than that of patients with difficult-to-control epilepsy ${ }^{27}$.

On the other hand, to omit the diagnosis of epilepsy may be just as damaging, or even more so. Patients with a diagnosis of PNES may be counseled to halt their AED use and reduce their visits to emergency healthcare facilities, so as to reduce iatrogenic levels and costs ${ }^{28,29}$. The treatment may be adapted to a condition of psychogenic nature ${ }^{30}$. Wyler et al. ${ }^{31}$ dramatically pointed out the possible consequences of these procedures when reporting the case of a 15-year-old girl who perished as a result of an ES that occurred after PNES had been diagnosed by means of VEEG and after medical discharge following AED withdrawal.

This prevalence of epilepsy among patients with PNES was $50 \%$ in an epidemiological study carried out in Iceland $d^{6}$, but in two recent studies ${ }^{12,16}$, relatively low frequencies of epilepsy were found, respectively 5.3 and $9.4 \%$. In our study, this association occurred in 14 patients (50\%), a high association level, even though our criteria for diagnosing epilepsy were more restrictive than in both of the abovementioned studies. As in these studies, epilepsy was defined as present when the patient presented ES during VEEG monitoring or otherwise, when unequivocal interictal epileptiform discharges were observed (sharp waves, spikes or complex spike-waves) although no ES occurred. Benign variants were not considered to be epileptiform conditions. However, unlike the two previous studies, interictal epileptiform discharges were considered to be present only when corroborated by clinical validation. Even if we had considered the presence of ES during VEEG monitoring as the one and only criterion for epilepsy, we would have had five patients (18\%) under these conditions, i.e. approximately twice the number of both previous studies, presenting relatively low rates of epilepsy.

Following the validation criteria of our study, we concluded that out of the 14 patients with epilepsy, eight (57.1\%) were in remission under treatment with AED or after AED withdrawal. Ramsay et al..$^{32}$ drew attention to the need to distinguish between simultaneous and sequential presence of epilepsy and PNES, when these conditions coexist. According to these authors, their simultaneous presence is easier to establish, due to the probable occurrence of ES during VEEG monitoring, whereas that may not happen when their occurrence is sequential. Usually, in these cases, existence of epilepsy precedes PNESD, complicates it and may, as these authors suggest, be related to the appearance of PNES.

PNES is usually considered to be present when the patient presents complete absence of therapeutic response to AED, or loss of response (therapeutic failure), or perhaps paradoxical responses to AED (worsening or spontaneous and unexpected remission). Moreover, it can occur eventually in function of atypical, multiple, inconsistent or changing pattern seizures, or when these are unleashed by an evident and specific stressful event with close timing connections with the occurrence of seizures ${ }^{33}$. The previous elements are particularly considered when the patient presents normal ancillary examinations (interictal routine EEGs and imaging studies such as CT, MRI and SPECT $)^{34,35}$. These situations lead the attentive physician to consider the possibility of referral to a center specializing in differential diagnosis and intensive monitoring by VEEG. Out of 22 patients from our sample who were referred for suspected PNES, nine (41\%) presented associated epilepsy. Out of six patients in our sample who were referred for other reasons, the presence of epilepsy was not confirmed or observed in only one of them. In these cases, the patients presented PNES as an unexpected phenomenon set within an investigative process directed towards other aims, such as pre-surgical evaluation or diagnostic evaluation of refractory epileptic syndromes. This draws attention to the importance of PNES as a clinical phenomenon that complicates the diagnostic process and treatment of patients with epilepsy.

In one of our cases, the patient presented both spontaneous and suggestion-induced CNEP during VEEG monitoring, but its occurrence was not validated by the medical history data (clinical validation), or by observation of video-recorded events by an external observer with close ties to the patient (observer validation). In this case, although PNES occurred during intensive monitoring by 
VEEG, we do not believe these represented a real clinical problem. The patient also presented complex partial epileptic seizures during intensive VEEG monitoring, originating from the frontal lobe, which was validated by both clinical and observer validation. This case points out the possibility of isolated PNES occurrence in some gullible individuals, especially when exposed to a favorable situation such as VEEG monitoring ${ }^{17}$. It also points out the risk of giving up the investigation far too early because of PNES occurrence, thereby leading to failure to diagnosed epilepsy.

As previously reported, although our criteria may be considered more restrictive than the two previous studies with relatively low rates of epilepsy, our findings suggest that the frequency of epilepsy in patients with PNES is much higher. What might be responsible for these differences? In these three studies, the populations received care in tertiary centers, with patients evaluated for similar reasons. Martin et al. ${ }^{16}$ drew attention to the size of their sample (514 patients with CNEP) and to a high rate of referral of patients for suspected PNES. However, out of 22 patients in our sample who were referred on these grounds, nine (41\%) presented associated epilepsy. This indicates that, even when the level of suspicion is high for PNES, coexistence of epilepsy may be a significant problem.

One significant difference between our study and others is the period of VEEG monitoring. In previous studies, evaluations typically lasted three days ${ }^{12,16}$, and did not exceed five days to one week. In our study, the typical duration of VEEG monitoring was three weeks, with a range from one to six weeks. What was the result of this prolonged monitoring? In most of our patients, PNES presented spontaneous or provoked occurrence, by induction with our protocol of suggestive techniques over the first days of evaluation. Epileptic seizures occurred generally some time after reduction or complete withdrawal of AEDs. In some cases, interictal discharges were registered only after AED withdrawal and a delayed observation period. Lengthening of the monitoring period was usually guided by medical history information, suggesting epilepsy as well as PNES. Possibly, early ending of VEEG monitoring after occurrence of PNES would lead to failure in the diagnosis of epilepsy.

In conclusion, our findings point out the need, at least in some cases, for prolonging the evaluation of patients with PNES and clinical histories indicating epilepsy.

\section{REFERENCES}

1. Benbadis SR, Lancman ME, King LM, Swanson SJ. Preictal pseudosleep: a new finding in psychogenic seizures. Neurology 1996;47:63-67.

2. Arnold, LM, Pritivera, MD. Psychopathology and trauma in epileptic and psychogenic seizure patients. Psychosomatics 1996:37:438-443.

3. Bowman ES. Pseudoseizures. Psychiatric Clin N Am 1998;21:649-657.

4. Sirven JL, Glosser DS. Psychogenic nonepileptic seizures: theoretic and clinical considerations. Neuropsychiatric Neuropsychol Behav Neurol 1998;11:225-235.
5. Benbadis SR, Allen Hauser W. An estimate of the prevalence of psychogenic non-epileptic seizures. Seizure 2000;9:280-281.

6. Sigurdardorttir KR, Olafsson E. Incidence of psychogenic seizures in adults: a population-based study in Iceland. Epilepsia 1998;39:749-752.

7. Szaflarski JP, Ficker DM, Cahill WT, Privitera MD. Four-year incidence of psychogenic nonepileptic seizures in adults in Hamilton County, OH. Neurology 2000;55:1561-1563.

8. Gates JR. Epidemiology and classification of non-epileptic events. In: Gates JR, Rowan AJ (Eds). Non-epileptic seizures. $2^{\text {nd }}$ ed. Boston: Butterworth-Heinemann, 2000:3-14.

9. Cragar DE, Berry DT, Fakhoury TA, Cibula JE, Schmitt FA. A review of diagnostic techniques in the differential diagnosis of epileptic and nonepileptic seizures. Neuropsychol Rev 2002;12:31-64.

10. LaFrance WC, Devinsky O. The treatment of psychogenic nonepileptic seizures: historical perspectives and future directions. Epilepsia 2004;45(Suppl 2):S15-S21.

11. Betts T, Boden S. Diagnosis, management and prognosis of a group of 128 patients with non-epileptic attack disorder. Part I. Seizure 1992;1:19-26.

12. Benbadis SR, Agrawal V, Tatum WO 4th. How many patients with psychogenic nonepileptic seizures also have epilepsy? Neurology 2001;57:915-917.

13. Devinsky O, Sanchez-Villasenor F, Vazquez B, Kothari M, Alper K, Luciano D. Clinical profile of patients with epileptic and nonepileptic seizures. Neurology 1996:46:1530-1533.

14. Kurcgant D, Marchetti RL, Marques AH, Marchetti LB. Crises pseudoepilépticas: diagnóstico diferencial. BJECN 2000;6:13-18.

15. Lesser RP, Lueders H, Dinner DS. Evidence for epilepsy is rare in patients with psychogenic seizures. Neurology 1983;33:502-504.

16. Martin R, Burneo JG, Prasad A, et al. Frequency of epilepsy in patients with psychogenic seizures monitored by video-EEG. Neurology 2003;61:1791-1792.

17. French J. The use of suggestion as a provocative test in the diagnosis of psychogenic non-epileptic seizures. In: Gates JR, Rowan AJ (Eds). Non-epileptic seizures. Boston: Butterworth-Heinemann, 1993:101-109.

18. Commission on Classification and Terminology of the International League Against Epilepsy. Proposal for revised clinical and electroencephalographic classification of epileptic seizures. Epilepsia 1981;22:489-501.

19. Commission on Classification and Terminology of the International League Against Epilepsy. Proposal for revised classification of epilepsies and epileptic syndromes. Epilepsia 1989;30:389-399.

20. Martin RC, Gilliam FG, Kilgore M, Faught E, Kuzniecky R. Improved health care resource utilization following video-EEG-confirmed diagnosis of nonepileptic psychogenic seizures. Seizure 1998;7:385-390.

21. Ettinger AB, Devinsky O, Weisbrot DM, Ramakrishna RK, Goyal A. A comprehensive profile of clinical, psychiatric, and psychosocial characteristics of patients with psychogenic nonepileptic seizures. Epilepsia 1999;40:1292-1298.

22. Walczak TS, Papacostas S, Williams DT, Scheuer ML, Lebowitz N, Notarfrancesco A. Outcome after diagnosis of psychogenic nonepileptic seizures. Epilepsia 1995;36:1131-1137.

23. Silva W, Giagante B, Saizar R, et al. Clinical features and prognosis of psychogenic non-epileptic seizures. Epilepsia 1991;40:263.

24. Betts T, Duffy N. Treatment of non-epileptic attack disorder (pseudoseizures) in the community. In: Gram L, Johannessen SI, Osterman PO, Sillanpaa M, (Eds). Pseudo-epileptic seizures. Briston: Wrightson Biomed Pub, 1993:109-121.

25. Niedermeyer E. The epilepsies: diagnosis and management. Baltimore: Urban \& Schwarzwnberg; 1990:251-256.

26. Lesley MA, Pritivera MD. Psychopathology and trauma in epileptic and psychogenic patients. Psychosomatics 1996;37:438-443.

27. Szaflarski JP, Hughes C, Szaflarski M, et al. Quality of life in psychogenic nonepileptic seizures. Epilepsia 2003;44:236-242.

28. Oto M, Espie C, Pelosi A, Selkirk M, Duncan R. The safety of antiepileptic drug withdrawal in patients with non-epileptic seizures. J Neurol Neurosurg Psychiatry 2005;76:1682-1685.

29. LaFrance WC Jr, Benbadis SR. Avoiding the costs of unrecognized psychological nonepileptic seizures. Neurology 2006;66:1620-1621.

30. McDade G, Brown SW. Non-epileptic seizures: management and predictive factors of outcome. Seizure 1992;1:7-10.

31. Wyler AR, Herman BP, Blumer D, Richey ET. In: Gates JR, Rowan AJ (Eds). Nonepileptic seizures. Boston: Butterworth-Heinemann, 1993:73-84.

32. Ramsay RE, Cohen A, Brown MC. Coexisting epilepsy and non-epileptic seizures. In: Gates JR, Rowan AJ, (eds). Non-Epileptic seizures. Boston: Butterworth-Heinemann, 1993:47-54.

33. Rowan AJ. Diagnosis of Non-Epileptic Seizures. In: Gates JR, Rowan AJ (Eds). Non-epileptic seizures. $2^{a}$ ed. Boston: Butterworth-Heinemann, 2000:15-30.

34. Davis BJ. Predicting nonepileptic seizures utilizing seizure frequency, EEG, and response to medication. Eur Neurol 2004;51:153-156.

35. Dworetzky BA, Strahonja-Packard A, Shanahan CW, Paz J, Schauble B, Bromfield EB. Characteristics of male veterans with psychogenic nonepileptic seizures. Epilepsia 2005;46:1418-1422. 